\section{A Useful New Endoscopic Marking Technique for Endoscopic Mucosal Resection of Early Gastric}

\section{Cancer}

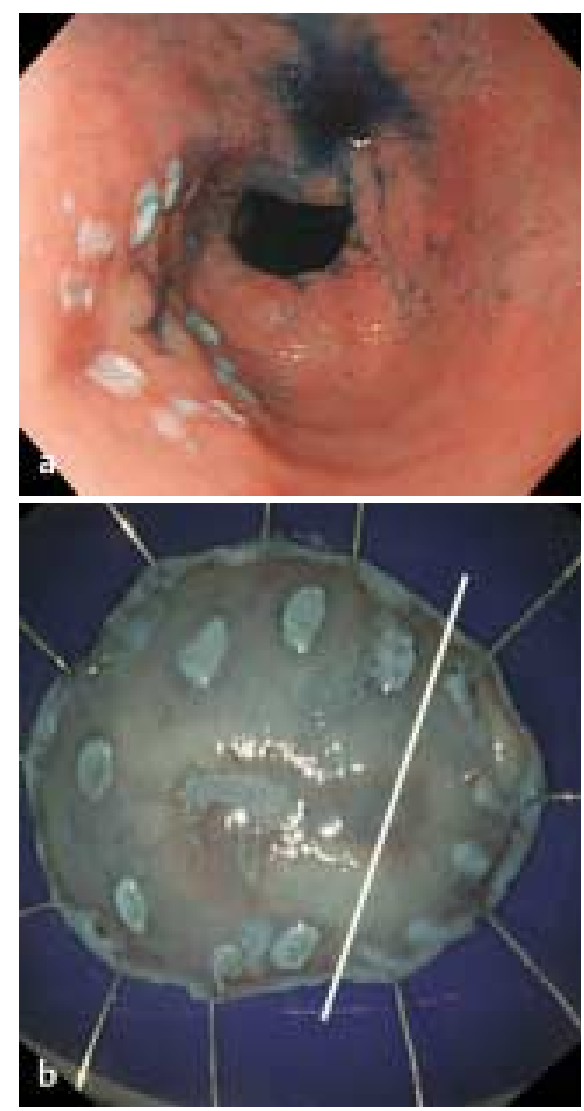

Figure 1 Markings around the $15 \mathrm{~mm}$ gastric cancer were made by APC on the distal side and by a needle knife on the proximal side, both giving quite a different macroscopic appearance ( $\mathbf{a}$ in vivo, $\mathbf{b}$ on the specimen).

Endoscopic mucosal resection (EMR) is a widely used and less invasive alternative to open surgery for early gastric cancer. The benefits include preservation of the stomach, noninvasiveness, and shorter hospital stays. In addition, the technique is indicated for lesions in which there is minimal risk of lymph-node involvement. Before the procedure, endoscopic marking of the tissue is very important to ensure a tumor-free margin and complete resection; in Japan, the marking is usually carried out with a needle-knife (Olympus KD-1L-1) [1]. The needle-knife is also used for precut procedures and mucosal incision for circumferential mucosal resection [2,3]. Argon plasma coagulation (APC) is a recently introduced therapeutic technique in the digestive tract, used to treat hemorrhagic and neoplastic lesions. Its advantages in-

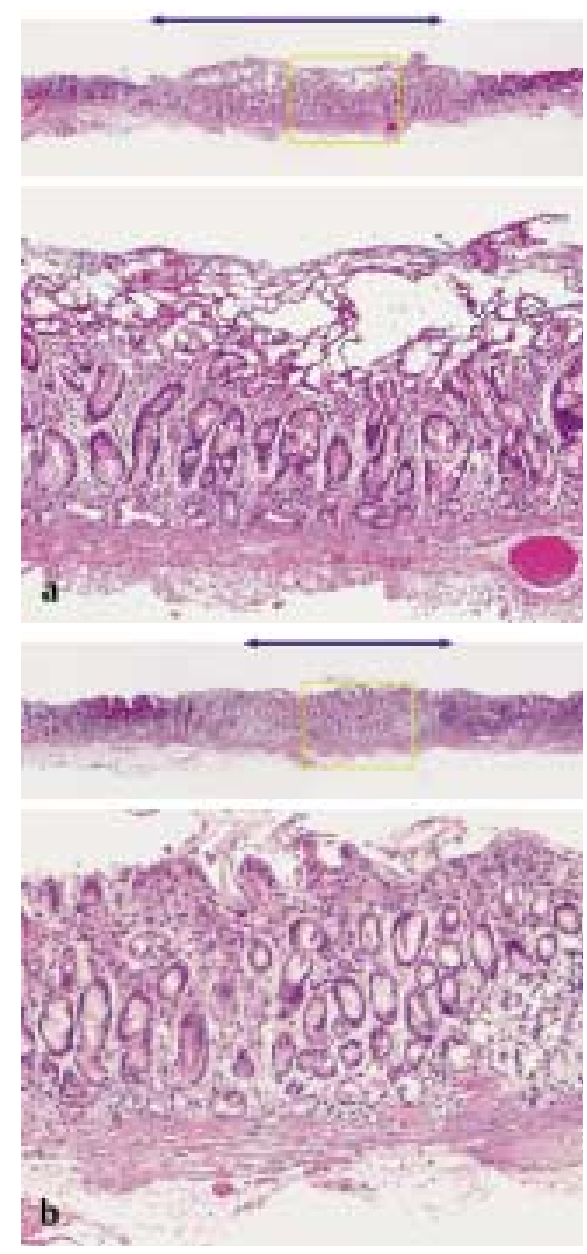

Figure 2 Histological differences between the two types of thermal injury: APC injury was limited to the mucosa and more uneven (a) than with the needle-knife (b).

clude effective and safe coagulation, controllable coagulation depth, a noncontact operating mode, and axial, radial, and retrograde application [4]. To avoid the risk of perforation during endoscopic marking with the needle-knife, we investigated the safety of APC and tested the use of the technique to create circumferential marks around early gastric cancer lesions before EMR. To the best of our knowledge, this is the first report of the use of APC for endoscopic marking before EMR. In a pilot study, we found that APC is safe and useful for carrying out endoscopic marking for EMR. In addition, APC combined with the use of a needle-knife also allows different types of mark to be made, making it much easier to differentiate the distal margin from the proximal margin in the retrieved resected specimens (Figures 1,2). Our group is currently conducting a prospective study to evaluate the safety and usefulness of this technique in a large number of patients. Research for this report was supported in part by a Grant-in-Aid for Cancer Research (12-4) from the Japanese Ministry of Health, Labor, and Welfare.

\section{H. Ooyanagi ${ }^{1}$, K.-I. Fu ${ }^{1}$, T. Ishikawa ${ }^{1}$,}

Y. Hirahara ${ }^{1}$, S. Igarashi ${ }^{2}$, T. Inada ${ }^{3}$

${ }^{1}$ Dept. of Diagnostic Imaging, Division of Endoscopy,

2 Dept. of Pathology,

${ }^{3}$ Dept. of Surgery, Tochigi Cancer Center Hospital, Utsunomiya, Tochigi, Japan.

\section{References}

${ }^{1}$ Hirao M, Masuda K, Asanuma T et al. Endoscopic mucosal resection of early gastric cancer and other tumors with local injection of hypertonic salineepinephrine. Gastrointest Endosc 1988; 34: 264-269

${ }^{2}$ Ono H, Kondo H, Gotoda T et al. Endoscopic mucosal resection for treatment of early gastric cancer. Gut 2001; 48: 225-229

${ }^{3}$ Yamamoto H, Kawata H, Sunada K et al. Success rate of curative endoscopic mucosal resection with circumferential mucosal incision assisted by submucosal injection of sodium hyaluronate. Gastrointest Endosc 2002; 56: 507-512

${ }^{4}$ Canard JM, Vedrenne B. Clinical application of argon plasma coagulation in gastrointestinal endoscopy: has the time come to replace the laser? Endoscopy 2001; 33: $353-357$

\section{Corresponding Author}

\section{K.-I. Fu, M.D.}

Dept. of Diagnostic Imaging, Division of Endoscopy Tochigi Cancer Center Hospital 4-9-13 Yohnan, Utsunomiya Tochigi 320-0834, Japan E-mail: kfuu@tcc.pref.tochigi.jp 